\title{
The Energy Dependent Pál-Bell Equation and the Influence of the Neutron Energy on the Survival Probability in a Supercritical Medium
}

\author{
J. E. M. Saxby ${ }^{1}$, M. M. R. Williams and M. D. Eaton \\ Nuclear Engineering Group, Department of Mechanical Engineering, Imperial College London, \\ Exhibition Road, London SW7 2AZ, UK \\ ${ }^{1}$ j.saxby12@imperial.ac.uk
}

\begin{abstract}
The survival probability of a neutron injected into a supercritical fissile medium is studied with respect to the energy dependence of the incoming neutron. We assume a point model but allow the energy dependence to be included through a general energy exchange model. We have studied the effect of slowing down on the survival probability by means of the Pál-Bell equation and the Goertzel-Greuling kernel, the separable kernel, the Hansen-Roach dataset and a WIMS generated dataset for a homogenised reactor to approximate the slowing down process. The Goertzel-Greuling model is known to be exact for $A=1$ and reverts to age theory for large mass ratios. It is also accurate for all intermediate mass numbers, except possibly when strong resonances are present. The separable kernel is a simple model of energy exchange, corresponding to the thermalisation of neutrons in a single collision, which ignores the slowing down process but provides a simple result allowing both ends of the reactor spectrum to be included. The Hansen-Roach data set is obtained from a realistic slowing down model in a fast system and the thermal system is modelled by homogenised reactor data, generated using WIMS, and is typical of the material found in a PWR. Using a scattering cross section which is an arbitrary function of energy, and capture and fission cross sections which are proportional to $1 / v$, we find that the survival probability is energy independent insofar as it depends only on the values of the ratio of the fission and capture cross sections. For non- $1 / v$ cross sections there is an energy dependence which we discuss below. The formalism developed is robust enough for studies to be made of the influence of resonance cross sections and inelastic scattering on survival probability.
\end{abstract}

\section{Introduction}

The survival probability of a neutron injected into a super-critical fissile medium has long been a subject of study in connection with low source start up (Hurwitz et al, 1963; Bell, 1963) and fast burst reactors such as Godiva (Wimmett et al, 1960; Hansen, 1960). A complete theory of this subject has been developed over the years, in particular by Bell (1965) and Pál $(1958,1962)$ and is summarised comprehensively 
in Pázsit and Pál (2008). The general theory encompasses a detailed energy, space and time dependence of the probability function but, in many practical cases, it is adequate to simplify this to the point model which is independent of space and energy. The point model assumes a global average over space, angle and energy and it is of some interest to examine the influence of the energy dependence of the cross sections and the slowing down process on the results obtained from it. These might be expected to be large as neutrons are born in fission in the $\mathrm{MeV}$ energy region and end up, after slowing down, in the thermal region $\sim 0.025 \mathrm{eV}$; thus a very large energy span is involved. We will study this matter by considering the survival probability of a neutron injected into an infinite medium at a given speed and examine the influence of the slowing down process on the outcome. For simplicity, we assume in one case that the material is pure ${ }^{235} U$ and in the other case that it is a homogeneous mixture of material typical of a PWR.

We will show that if the absorption and fission cross sections follow the $1 / v$ law, then the survival probability is independent of the slowing down model. For more realistic variations of cross section energy dependence, the survival probability is found to be a function of the slowing down model. Some explicit examples of this behaviour are given by developing a numerical method using three different models for the scattering process: Goertzel-Greuling, separable kernel (Williams, 1966) and finally the use of explicit scattering cross sections from both the Hansen and Roach dataset (1961) for a fast system and a WIMS generated dataset for a thermal system.

\section{General Theory}

\subsection{Energy dependent Pál-Bell equation and its influence on the point model results}

The complete Pál-Bell equation, as derived by Pázsit and Pál (2008), for the generating function

$$
G\left(z, t, R \mid \overrightarrow{r_{0}}, \overrightarrow{v_{0}}, s\right)=\sum_{n=0}^{\infty} z^{n} P\left(n, t, R \mid \overrightarrow{r_{0}}, \overrightarrow{v_{0}}, s\right)
$$

is given by

$$
\begin{aligned}
\frac{\partial G\left(z, t, R \mid \overrightarrow{r_{0}}, \overrightarrow{v_{0}}, s\right)}{\partial s}+\hat{T} G\left(z, t, R \mid \overrightarrow{r_{0}}, \overrightarrow{v_{0}}, s\right)+\lambda_{f}\left(\overrightarrow{r_{0}}, \overrightarrow{v_{0}}, s\right) f\left[G_{p}\left(z, t, R \mid \overrightarrow{r_{0}}, s\right), \overrightarrow{v_{0}}\right] \\
+\lambda_{c}\left(\overrightarrow{r_{0}}, \overrightarrow{v_{0}}, s\right)=0
\end{aligned}
$$

where $\lambda_{a}=\lambda_{c}+\lambda_{f}$

$$
\begin{aligned}
\hat{T} G\left(z, t, R \mid \overrightarrow{r_{0}}, \overrightarrow{v_{0}}, s\right)= & \left(\lambda_{a}\left(\overrightarrow{r_{0}}, \overrightarrow{v_{0}}, s\right)+\lambda_{s}\left(\overrightarrow{r_{0}}, \overrightarrow{v_{0}}, s\right)\right) G\left(z, t, R \mid \overrightarrow{r_{0}}, \overrightarrow{v_{0}}, s\right) \\
& +\overrightarrow{v_{0}} \cdot \nabla \overrightarrow{r_{0}} G\left(z, t, R \mid \overrightarrow{r_{0}}, \overrightarrow{v_{0}}, s\right) \\
& +\lambda_{s}\left(\overrightarrow{r_{0}}, \overrightarrow{v_{0}}, s\right) \int d \vec{v}^{\prime} g\left(\overrightarrow{v_{0}} \rightarrow \overrightarrow{v^{\prime}}\right) G\left(z, t, R \mid \overrightarrow{r_{0}}, \overrightarrow{v^{\prime}}, s\right)
\end{aligned}
$$

Where $g\left(\overrightarrow{v_{0}} \rightarrow \vec{v}^{\prime}\right)$ is the slowing down kernel and 


$$
G_{p}\left(z, t, R \mid \overrightarrow{r_{0}}, s\right)=\int d{\overrightarrow{v_{0}}}^{\prime} F_{0}\left(\overrightarrow{v_{0}}\right) G\left(z, t, R \mid \overrightarrow{r_{0}},{\overrightarrow{v_{0}}}^{\prime}, s\right)
$$

$f\left(x, \overrightarrow{v_{0}}\right)$ defines the random emission of neutrons in a fission event and is defined by

$$
f\left(x, \overrightarrow{v_{0}}\right)=\sum_{k=0}^{K} \frac{(-1)^{k}}{k !} \chi_{k}\left(\overrightarrow{v_{0}}\right)(1-x)^{k}
$$

with the multiplicity $\chi_{k}$, given in terms of the probability of $\nu$ neutrons emitted in a fission event $p_{\nu}$, by

$$
\chi_{k}=\sum_{\nu=k}^{K} \frac{\nu !}{(\nu-k) !} p_{\nu}
$$

Note that we use slightly different notation to Pázsit and Pál in that they use $Q$ to denote the transition rate and we use $\lambda$. Also, Pázsit and Pál use $Q_{a}$ for their capture rate while we use $\lambda_{c}$, with $\lambda_{a}=\lambda_{c}+\lambda_{f} \cdot F_{0}(\vec{v})$ is the fission spectrum of the prompt neutrons. The final conditions associated with equation 2 are

$$
G\left(z, t, R \mid \overrightarrow{r_{0}}, \overrightarrow{v_{0}}, t\right)=1-(1-z) \Delta\left(\overrightarrow{r_{0}}, V_{r}\right) \Delta\left(\overrightarrow{v_{0}}, U_{v}\right)
$$

where

$$
\Delta\left(\overrightarrow{u_{0}}, U\right)=1 \text { if } \overrightarrow{u_{0}} \in U \text { and } \Delta\left(\overrightarrow{u_{0}}, U\right)=0 \text { if } \overrightarrow{u_{0}} \notin U
$$

$G\left(z, t, R \mid \overrightarrow{r_{0}}, \overrightarrow{v_{0}}, s\right)$, as defined above is the 'single particle generating function' and is always time dependent as it relates to the chain initiated by a single neutron. $V_{r}$ is a sub-region within the reactor and $U_{v}$ is an energy range; generally the complete range. The usefulness of $G$ is that it does not change when different sources are used and, in a loose sense, is analogous to a Greens function. To relate $G$ to the case where there is an independent source, we proceed as follows. Such a source can itself emit varying numbers of neutrons at each disintegration and this will also be dealt with in the same way as for the forward equation. Now if the source emits neutrons with a compound Poisson distribution with varying multiplicity, we may write for the source generating function (Bartlett, 1955)

$$
\begin{aligned}
G_{S}(z, t, R \mid s)=\exp & {\left[\int_{s}^{t} d s^{\prime} \int_{V_{\vec{r}}} d \vec{r} \int_{U_{\vec{v}}} d \vec{v} S_{d}\left(\vec{r}, \vec{v}, s^{\prime}\right)\left[f_{q}\left(G\left(z, t, R \mid \vec{r}, \vec{v}, s^{\prime}\right)\right)-1\right]\right] } \\
& =\sum_{N=0}^{\infty} z^{N} p_{s}(N, t \mid s)
\end{aligned}
$$

with $f_{q}(z)$ the generating function for the source emission and $S_{d}$ the disintegration rate of the process that leads to the source neutrons. Note that while $G$ is always time 
dependent $G_{S}$ with be asymptotically time-independent for a sub-critical system. The equation for $G_{S}$ can also be written

$$
-\frac{\partial G_{S}(z, t, R \mid s)}{\partial s}=\int_{V_{\vec{r}}} d \vec{r} \int_{U_{\vec{v}}} d \vec{v} S_{d}(\vec{r}, \vec{v}, s)\left[f_{q}(G(z, t, R \mid \vec{r}, \vec{v}, s))-1\right] G_{S}(z, t, R \mid s)
$$

We will not be concerned with an independent source but cite the above generalisation for completeness.

Although the Pál-Bell equation is given in terms of $G\left(z, t, R \mid \overrightarrow{r_{0}}, \overrightarrow{v_{0}}, s\right)$, it is usually more convenient in practice to use the modified generating function $\tilde{G}\left(z, t, R \mid \overrightarrow{r_{0}}, \overrightarrow{v_{0}}, s\right)=$ $1-G\left(z, t, R \mid \overrightarrow{r_{0}}, \overrightarrow{v_{0}}, s\right)$. The reason for this is evident from equation 5 , which is a rapidly converging series for many practical situations. Indeed, taking only terms up to $k=2$, leads to the well-known and accurate quadratic approximation.

For the problem under consideration, we are only interested in the infinite medium time dependent case. In this instance we may neglect the spatial operator and consider an isotropic distribution of neutrons. We also assume that the cross sections themselves are time independent which leads to

$$
\begin{gathered}
\tilde{G}(z, t \mid v, s) \rightarrow \tilde{G}(z, v, t) \\
{\left[\frac{1}{v} \frac{\partial}{\partial t}+\Sigma(v)\right] \tilde{G}(z, v, t)=} \\
\Sigma_{s}(v) \int_{0}^{\infty} d v^{\prime} g\left(v \rightarrow v^{\prime}\right) \tilde{G}\left(z, v^{\prime}, t\right)-\Sigma_{f}(v) \sum_{k=1}^{K} \frac{(-1)^{k}}{k !} \chi_{k}\left[\int_{0}^{\infty} d v^{\prime} F\left(v^{\prime}\right) \tilde{G}\left(z, v^{\prime}, t\right)\right]^{k}
\end{gathered}
$$

where $\Sigma=\Sigma_{a}+\Sigma_{s}, \Sigma_{a}=\Sigma_{c}+\Sigma_{f}$ and $F(v)$ is the fission spectrum. The initial condition is $\tilde{G}(z, v, 0)=1-z$. It is convenient to convert this equation to the energy variable, where we have

$$
\left(\frac{1}{v} \frac{\partial}{\partial t}+\Sigma(E)\right) \tilde{G}(z, E, t)=\Sigma_{s}(E) \int_{0}^{\infty} d E^{\prime} g\left(E \rightarrow E^{\prime}\right) \tilde{G}\left(z, E^{\prime}, t\right)+\Sigma_{f}(E) f(\zeta(z, t))
$$

with

$$
f(\zeta)=-\sum_{k=1}^{K} \frac{(-1)^{k}}{k !} \chi_{k} \zeta^{k} \quad \zeta(z, t)=\int_{0}^{\infty} d E^{\prime} F\left(E^{\prime}\right) \tilde{G}\left(z, E^{\prime}, t\right)
$$

Note that the linear part of equations 12 and 13 is the adjoint of the normal slowing down equation. 
Let us multiply by $v$ and re-write equation 13 as

$$
\left(\frac{\partial}{\partial t}+v \Sigma(E)\right) \tilde{G}(z, E, t)=v \Sigma_{s}(E) \int_{0}^{\infty} d E^{\prime} g\left(E \rightarrow E^{\prime}\right) \tilde{G}\left(z, E^{\prime}, t\right)+v \Sigma_{f}(E) f(\zeta(z, t))
$$

Suppose the absorption and fission cross sections are proportional to $1 / v$ so that we have $v \Sigma_{a}(E)=\lambda_{a}, v \Sigma_{f}(E)=\lambda_{f}$ and $v \Sigma_{s}(E)=\lambda_{s}(E)$ where $\lambda_{s}(E)$ is an arbitrary function of $E$. Then 15 becomes

$$
\left(\frac{\partial}{\partial t}+\lambda_{a}+\lambda_{s}(E)\right) \tilde{G}(z, E, t)=\lambda_{s}(E) \int_{0}^{\infty} d E^{\prime} g\left(E \rightarrow E^{\prime}\right) \tilde{G}\left(z, E^{\prime}, t\right)+\lambda_{f} f(\zeta(z, t))
$$

Suppose we now assume that $\tilde{G}(z, E, t)=\theta(z, t)$ and insert this into equation 16 . We then find

$$
\left(\frac{\partial}{\partial t}+\lambda_{a}+\lambda_{s}(E)\right) \theta(z, t)=\lambda_{s}(E) \theta(z, t) \int_{0}^{\infty} d E^{\prime} g\left(E \rightarrow E^{\prime}\right)+\lambda_{f} f(\zeta(z, t))
$$

But we know that $\int_{0}^{\infty} d E^{\prime} g\left(E \rightarrow E^{\prime}\right)=1$, so the scattering terms cancel and we get

$$
\left(\frac{\partial}{\partial t}+\lambda_{a}\right) \theta(z, t)=\lambda_{f} f(\zeta(z, t))
$$

But

$$
\zeta(z, t)=\int_{0}^{\infty} d E^{\prime} F\left(E^{\prime}\right) \tilde{G}\left(z, E^{\prime}, t\right)=\theta(z, t) \int_{0}^{\infty} d E^{\prime} F\left(E^{\prime}\right)=\theta(z, t)
$$

hence equation 18 becomes

$$
\left(\frac{\partial}{\partial t}+\lambda_{a}\right) \zeta(z, t)=\lambda_{f} f(\zeta(z, t))
$$

Although the above is not a rigorous proof in the mathematical sense, it strongly suggests that for $1 / v$ absorption cross sections the survival probability $\zeta(0, t)$ is independent of the slowing down model. Numerical work will confirm the above assumption. An analogous result arises if delayed neutrons are included. For the asymptotic value, as $t \rightarrow \infty$, we find the following equation for survival probability.

$$
\lambda_{a} \zeta=\lambda_{f} f(\zeta)
$$

We now consider the asymptotic value of the survival probability, viz $\zeta(0, \infty)$, in which case $\partial \tilde{G} / \partial t=0$ and we arrive at the equation for the energy dependence of $\tilde{G}(0, E, \infty)=\theta(E)$ 


$$
\Sigma(E) \theta(E)=\Sigma_{s}(E) \int_{0}^{\infty} d E^{\prime} g\left(E \rightarrow E^{\prime}\right) \theta\left(E^{\prime}\right)+\Sigma_{f}(E) f(\zeta)
$$

Remember for the $1 / v$ cases $\theta(E)$ was independent of energy, but for non $1 / v$ models $\theta(E)$ should depend on energy. This is in line with expectation as for $1 / v$ capture and fission cross sections the transition probability per unit time is constant. As the process is Markovian, and there is no leakage, this is irrespective of scattering events thus the slowing down history can have no effect. We now go on to consider some analytical solutions to confirm our proof regarding $1 / v$ cross sections.

\subsection{Analytical Solution in the Slowing Down Range}

It is convenient to use the lethargy variable for this case where $u=\ln \left(E_{0} / E\right)$. To proceed, we assume that slowing down is by elastic collisions and for the slowing down kernel, $g\left(u \rightarrow u^{\prime}\right)$, we will take the Goertzel-Greuling approximation. This has the attribute that it is exact for $A=1$ and $A>>1$ and is also a very good approximation for all $A$ values (Williams, 1966). The form of $g\left(u \rightarrow u^{\prime}\right)$ is given by

$$
g\left(u \rightarrow u^{\prime}\right)=\frac{\xi}{\gamma^{2}} e^{\left(u-u^{\prime}\right) / \gamma} H\left(u^{\prime}-u\right)+\left(1-\frac{\xi}{\gamma}\right) \delta\left(u-u^{\prime}\right)
$$

where $H(x)$ is the Heaviside function and the slowing down parameters $\xi$ and $\gamma$ are given by

$$
\begin{gathered}
\xi=1+\frac{\alpha}{1-\alpha} \ln (\alpha) \quad \gamma=\frac{\bar{a}}{\xi} \quad \bar{a}=1-\frac{\alpha}{1-\alpha} q\left(1+\frac{q}{2}\right) \\
\alpha=\left(\frac{A-1}{A+1}\right)^{2} \quad q=-\ln (\alpha)
\end{gathered}
$$

Inserting 23 into 15 leads to

$$
\left(\frac{\gamma}{v} \frac{\partial}{\partial t}+\gamma \Sigma_{a}(u)+\xi \Sigma_{s}(u)\right) \tilde{G}(z, u, t)=\frac{\xi}{\gamma} \Sigma_{s}(u) \int_{u}^{\infty} d u^{\prime} \tilde{G}\left(z, u^{\prime}, t\right) e^{\left(u-u^{\prime}\right) / \gamma}+\gamma f(\zeta) \Sigma_{f}(u)
$$

setting $z=0$ and allowing $t \rightarrow \infty$ we find, after considerable algebra with $v \Sigma_{f}=$ $v_{0} \Sigma_{f 0}$ and $v \Sigma_{a}=v_{0} \Sigma_{c 0}+v_{0} \Sigma_{f 0}$, that

$$
\zeta=\Lambda\left(\bar{\nu} \zeta-\sum_{k=2}^{K} \frac{(-1)^{k}}{l !} \chi_{k} \zeta^{k}\right)
$$

Where $\Lambda=\lambda_{f} /\left(\lambda_{f}+\lambda_{c}\right)$. To find $\theta(E)$ we use the steady state of equation 25 and obtain the energy variable

$$
\theta(E)=\Lambda\left(\bar{\nu} \zeta-\sum_{k=2}^{K} \frac{(-1)^{k}}{k !} \chi_{k} \zeta^{k-1}\right)=\zeta
$$


which depends only on the cross sections evaluated at the energy of injection.

As far as the thermal region is concerned, there is a simple model of energy exchange called the separable kernel (Williams, 1966) which can be written

$$
g\left(E \rightarrow E^{\prime}\right)=\frac{1}{\bar{\Sigma}_{s}} M\left(E^{\prime}\right) \Sigma_{s}\left(E^{\prime}\right)
$$

where

$$
M\left(E^{\prime}\right)=\frac{E^{\prime}}{T^{2}} e^{-E^{\prime} / T} \quad \bar{\Sigma}_{s}=\int_{0}^{\infty} d E^{\prime} M\left(E^{\prime}\right) \Sigma_{s}\left(E^{\prime}\right)
$$

$T$ is the physical temperature in the medium in eV. Physically this model corresponds to thermalisation in one collision and ignores the actual slowing down process, nevertheless it does lead to a simple result which enables both ends of the reactor spectrum to be included. Use of this kernel in 16 leads to the result in 27 and confirms the proof in section 2.1.

\section{Numerical Solutions}

\subsection{The Goertzel-Greuling Kernel}

We now wish to consider the numerical solution of equation 22 with the GoertzelGreuling kernel via a six group energy discretisation for $\theta$. We firstly discretise equation 22 to give

$$
\Sigma_{t g} \theta_{g}-\Sigma_{s g} \sum_{g^{\prime}}^{G} g_{g^{\prime} \rightarrow g} \theta_{g^{\prime}}=-\Sigma_{f g} \sum_{k=1}^{K} \frac{(-1)^{k}}{k !} \chi_{k}\left[\sum_{g^{\prime}}^{G} F_{g^{\prime}} \theta_{g^{\prime}}\right]^{k}
$$

with

$$
g\left(E \rightarrow E^{\prime}\right)=\frac{\xi}{\gamma^{2}}\left(\frac{E^{\prime}}{E}\right)^{\frac{1}{\gamma}}+\left(1-\frac{\xi}{\gamma}\right) \delta\left(E-E^{\prime}\right)
$$

which, when discretised, gives

$$
g_{g \rightarrow g^{\prime}}=\frac{\xi}{\gamma^{2}}\left(\frac{E_{g^{\prime}}}{E_{g}}\right)^{\frac{1}{\gamma}}+\left(1-\frac{\xi}{\gamma}\right) \delta_{g, g^{\prime}}
$$

Where $E_{g}$ is the group energy. Thus giving us the full discretised equation to be solved as

$$
\Sigma_{t g} \theta_{g}-\Sigma_{s g} \sum_{g^{\prime}}^{G}\left(\frac{\xi}{\gamma^{2}}\left(\frac{E_{g^{\prime}}}{E_{g}}\right)^{\frac{1}{\gamma}}+\left(1-\frac{\xi}{\gamma}\right) \delta_{g, g^{\prime}}\right) \theta_{g^{\prime}}=-\Sigma_{f g} \sum_{k=1}^{K} \frac{(-1)^{k}}{k !} \chi_{k}\left[\sum_{g^{\prime}}^{G} F_{g^{\prime}} \theta_{g^{\prime}}\right]^{k}
$$


To simplify the solution of this equation, we divide through by $f(\zeta)$ as given in equation 14 to give

$$
\Sigma_{t g} \hat{\theta}_{g}-\Sigma_{s g} \sum_{g^{\prime}}^{G}\left(\frac{\xi}{\gamma^{2}}\left(\frac{E_{g^{\prime}}}{E_{g}}\right)^{\frac{1}{\gamma}}+\left(1-\frac{\xi}{\gamma}\right) \delta_{g, g^{\prime}}\right) \hat{\theta}_{g^{\prime}}=-\Sigma_{f g}
$$

which is simply a set of linear equations in $\hat{\theta}_{g}$ which can be solved using a routine from the linear algebra package LAPACK (Anderson et al, 1999). From here it is simple to calculate $\Lambda$ via

$$
\sum_{g^{\prime}=1}^{G} F_{g^{\prime}} \hat{\theta}_{g^{\prime}}=\Lambda
$$

The survival probability, $\zeta_{0}$ is then found as the root in the range $(0,1)$ of

$$
\zeta=\Lambda f(\zeta)
$$

Finally, $\theta$ can be recovered via

$$
\theta_{g}=\hat{\theta}_{g} f(\zeta)
$$

We investigate this expression for $A=1$, where the Goertzel-Greuling model is known to be exact, using capture and fission cross sections given by

$$
\Sigma_{c}(E)=\Sigma_{c 0}\left(\frac{E_{0}}{E}\right)^{1 / 2} \text { and } \Sigma_{f}(E)=\Sigma_{f 0}\left(\frac{E_{0}}{E}\right)^{1 / 2}
$$

Both $\Sigma_{s}$ independent of energy and $\Sigma_{s}$ in the same form as the cross sections in equation 38 were considered for six energy groups, with energies provided for pure ${ }^{235} U$ by Hansen and Roach (1961) and using a reference energy of $E_{0}=4.41 \mathrm{MeV}$ to fix the values of $v \Sigma_{f}(E)$ and $v \Sigma_{c}(E)$. The value of $E$ in $\theta(E)$ is the energy of the initiating neutron.

\begin{tabular}{l|cccccc|c}
$\Sigma_{s}$ & $\theta_{1}$ & $\theta_{2}$ & $\theta_{3}$ & $\theta_{4}$ & $\theta_{5}$ & $\theta_{6}$ & $\Lambda$ \\
\hline constant & 0.899335 & 0.899335 & 0.899335 & 0.899335 & 0.899335 & 0.899335 & 0.960317 \\
$\propto \frac{1}{v}$ & 0.899335 & 0.899335 & 0.899335 & 0.899335 & 0.899335 & 0.899335 & 0.960317
\end{tabular}

Table 1: Survival probabilities for $1 / v$ cross sections and Goertzel-Greuling kernel for $A=1$. 
As can be seen, the results are constant across the energy groups, being consistent to 14 significant figures. We can also consider the above for $A=2$, where the Goertzel-Greuling model is considered to be least accurate. Under these conditions, we find exactly the same results as in table 1 and confirms quantitatively our assertion that the survival probability is independent of the slowing down model.

It is informative to consider how this result may vary if instead of using six energy groups we used just one, with energy averaged cross sections. In this case, a result of $\theta=0.899335$ was found, which was consistent to the results found above to seven significant figures, $5.1 \times 10^{-7 \%}$. We can also compare this to the analytical solution given in equation 27. Evaluating this with the same data as in the code gives $\theta=0.899335$, which agrees with the results given previously. It should be noted that because the ratio of fission to absorption is high, 0.960317, the system is very highly multiplying with an infinite medium multiplication factor of about 2.3. For this reason all the multiplicities, $\chi_{k}$ in the expansion $f(\zeta)$ are needed and the quadratic approximation, where terms of up to $\chi_{2}$ are kept, is not valid.

From the theory developed in Section 2, we expect the survival probability to depend on the value of the transition rates $\lambda_{f}=v \Sigma_{f}$ and $\lambda_{c}=v \Sigma_{c}$ corresponding to the fission and capture cross section respectively. For convenience we have used the values for $\Sigma_{c 0}, \Sigma_{f 0}$ and $E_{0}$ in equation 38 from the Hansen and Roach dataset to illustrate the variation in the capture and fission cross sections at different energies. The results of this are shown in figure 1 and are as expected in view of equation 38 where we expect the survival probability to increase according to the ratio $\Lambda\left(E_{0}\right)=\lambda_{f} /\left(\lambda_{f}+\lambda_{c}\right)$. The figure indicates that the survival probability is tending to a limiting value. This may be explained by examining the values of $\Lambda$ for each group. We find that the ratio $\Lambda$ takes the values $0.796,0.861,0.889,0.917,0.938$ and 0.960 as the energy increases. Thus the survival probability increases with energy, as confirmed by our results in figure 1 .

It is a fair question to ask what the average survival probability is if the medium is irradiated with neutrons with a spectrum of energies $S(E)$; for example if the source were from polonium-beryllium reactions. In this case, the statistics of the source neutrons must be included and the use of equation 9 for $G_{S}$. However we do not consider that here as we are only concerned with the first persistent chain initiated by a single neutron.

\subsection{The Separable Kernel}

The discretised version of the kernel in equation 28 can be written

$$
g_{g \rightarrow g^{\prime}}=\frac{1}{\bar{\Sigma}_{s}} M_{g^{\prime}} \Sigma_{s g^{\prime}}
$$

with 29 becoming

$$
M_{g^{\prime}}=\frac{E_{g^{\prime}}}{T^{2}} e^{-E_{g^{\prime}} / T} \quad \bar{\Sigma}_{s}=\sum_{g^{\prime}}^{G} M_{g^{\prime}} \Sigma_{s g^{\prime}}
$$




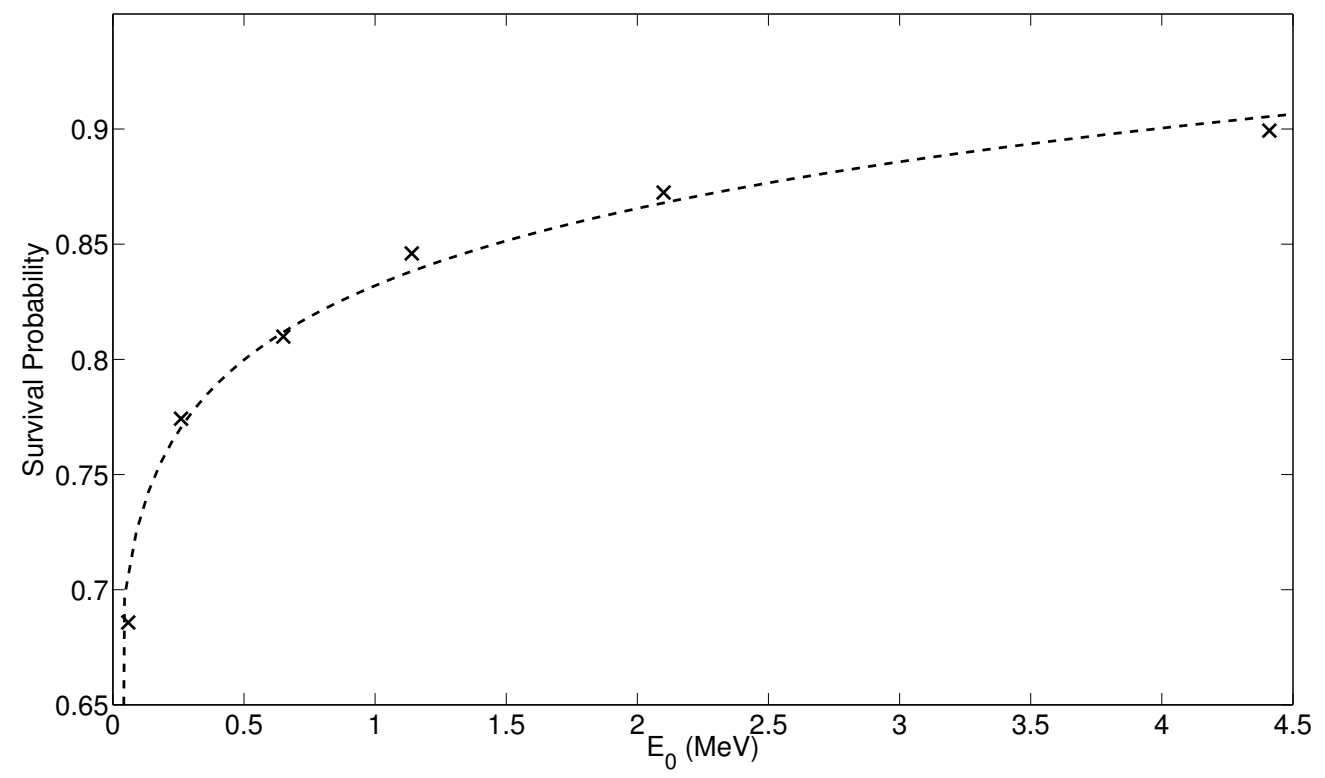

Figure 1: Variation in survival probability for different values of $E_{0}$ which set $\lambda_{f}$ and $\lambda_{c}$ according to the Hansen and Roach group structure.

Inserting equation 39 into equation 30 the full discretised equation to be solved is

$$
\Sigma_{t g} \theta_{g}-\Sigma_{s g} \sum_{g^{\prime}}^{G} \frac{1}{\bar{\Sigma}_{s}} M_{g^{\prime}} \Sigma_{s g^{\prime}} \theta_{g^{\prime}}=-\Sigma_{f g} \sum_{k=1}^{K} \frac{(-1)^{k}}{k !} \chi_{k}\left[\sum_{g^{\prime}}^{G} F_{g^{\prime}} \theta_{g^{\prime}}\right]^{k}
$$

The numerical results from the above equations are identical to those in Table 1 and again confirm our assertion regarding $1 / \mathrm{v}$ cross sections and the slowing down model.

\subsection{Hansen and Roach Cross Section Data}

We now consider the scattering cross sections given in Hansen and Roach (1961). Keeping the capture and fission cross sections in the form of equation 38 (i.e. $1 / v$ ), but using explicit values for $\Sigma_{s g \rightarrow g^{\prime}}$ allows us to write equation 30 as

$$
\Sigma_{t g} \theta_{g}-\sum_{g^{\prime}}^{G} \Sigma_{s g \rightarrow g^{\prime}} \theta_{g^{\prime}}=-\Sigma_{f g} \sum_{k=1}^{K} \frac{(-1)^{k}}{k !} \chi_{k}\left[\sum_{g^{\prime}}^{G} F_{g^{\prime}} \theta_{g^{\prime}}\right]^{k}
$$

This can be solved in the same manner as section 3.1. Implementing such a solution gives the results shown in Table 2, which are consistent between groups, and are also consistent with results in the previous sections to 14 significant figures.

It is also interesting to consider how these results might vary if instead of using the $1 / v$ fission and capture cross sections, we used the Hansen and Roach data set for all cross sections. In this case, we find the results shown in table 3. The survival probabilities now depend on energy, but not strongly. As before, it is possible to use 


\begin{tabular}{l|cccccc|c}
$\Sigma_{s}$ & $\theta_{1}$ & $\theta_{2}$ & $\theta_{3}$ & $\theta_{4}$ & $\theta_{5}$ & $\theta_{6}$ & $\Lambda$ \\
\hline constant & 0.899335 & 0.899335 & 0.899335 & 0.899335 & 0.899335 & 0.899335 & 0.960317
\end{tabular}

Table 2: Survival probabilities for $1 / v$ cross sections and Hansen and Roach energy transfer.

the one group model, with average cross sections. In this case we find $\theta=0.815907$ which is a not unreasonable representative value, and is extremely close to the survival probability averaged over the fission spectrum of $\theta=0.815908$.

\begin{tabular}{l|cccccc|c}
$\Sigma_{s}$ & $\theta_{1}$ & $\theta_{2}$ & $\theta_{3}$ & $\theta_{4}$ & $\theta_{5}$ & $\theta_{6}$ & $\Lambda$ \\
\hline constant & 0.726737 & 0.783818 & 0.802343 & 0.816870 & 0.824603 & 0.832695 & 0.893577
\end{tabular}

Table 3: Survival probabilities for Hansen and Roach cross sections and energy transfer.

\subsection{Almenas Homogenised Reactor Test Case}

Finally, we wish to consider the variation of survival probability with energy in a homogenised reactor core operating at $250^{\circ} \mathrm{C}$ with a water density of $0.8 \mathrm{~g} / \mathrm{cm}^{3}$. The composition of the core we wish to investigate is given in Almenas (1992) as

\begin{tabular}{l|c|c|c|c} 
reactor material & $\mathrm{UO}_{2}$ & $\mathrm{Zr}$ & $\mathrm{Fe}$ & $\mathrm{H}_{2} \mathrm{O}$ \\
\hline percentage composition & 32 & 8 & 2 & 58
\end{tabular}

Table 4: Volume percentage composition of reactor core, reproduced from Almenas \& Lee (1992)

In order to calculate the survival probability for such a reactor, macroscopic cross sections were generated for the 172 group case using the Winfrith Improved Multigroup Scheme (WIMS) (Amec Foster Wheeler, 2014). To achieve this, a calculation was performed for 1-D cylindrical geometry with white reflective boundary conditions, yielding an infinite homogeneous configuration using the THESEUS 1-D collision probability module of WIMS (Jonsson, 1963). The 172 energy group XMAS library in WIMS was used as the base nuclear data library for the calculations and was generated from the JEF2.2 nuclear data library (Nuclear Energy Agency, 2000) using the NJOY nuclear processing code (MacFarlane et al, 2012). Resonance self-shielding was performed within the WIMS calculations using sub-group resonance self-shielding methods (Roth, 1974).

Once the cross sections were generated, the survival probability for each group could be calculated using the same method as detailed in section 3.3. The result of implementing such a solution is shown in figure 2 .

As can be seen from figure 2, when detailed cross sections are used, the survival probability varies significantly with neutron energy. The survival probability using a one group average cross section is $\zeta=0.190668$, and is the same as the average over the fission spectrum of the 172 energy group survival probabilities. 


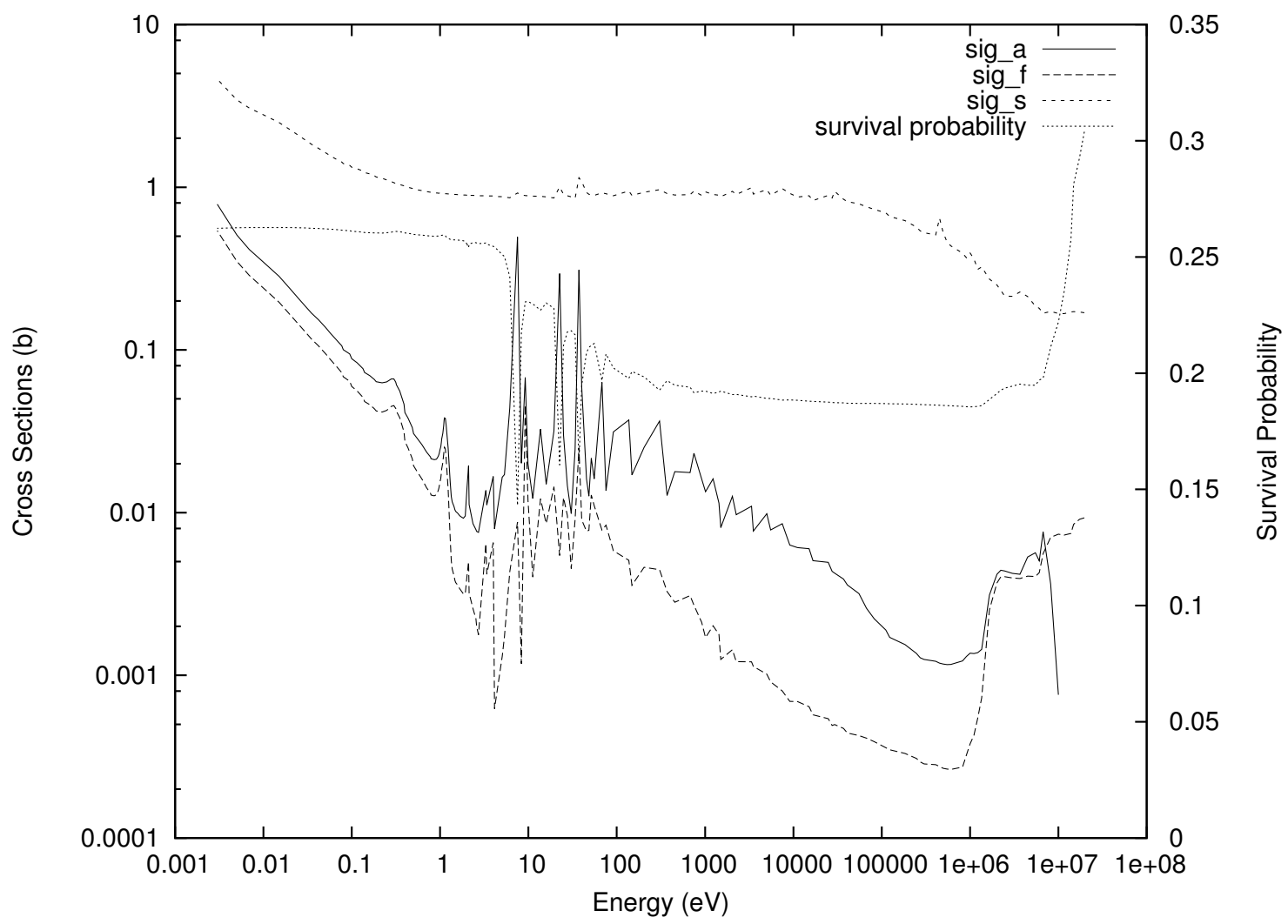

Figure 2: Variation in survival probability on the right hand axis, and scattering, absorption and fission cross sections on the left hand axis with neutron energy for a homogenised reactor (Almenas \& Lee, 1992).

When the quadratic approximation is used, i.e. keeping terms up to $k=2$ in equation 14, the one-group survival probability is modified to $\zeta=0.171935$ and the average survival probability in the 172 group case is $\zeta=0.172748$. These figures show that in the case of the thermal system, which is far less multiplying than the fast Hansen-Roach system, the quadratic approximation is a reasonable approximation.

\section{Conclusions}

We note, from the above work, that the slowing down and thermalisation properties of the medium do not affect the value of the survival probability if the capture and fission cross sections follow the $1 / v$ law. This has been shown analytically and confirmed numerically for the Goertzel-Greuling kernel, the separable kernel and the Hansen-Roach energy transfer rates. This also implies, that for $1 / v$ cross sections, the survival probability is constant with initiating energy and depends only on the ratio $\Lambda$. However, when the full cross section data set is considered via the Hansen-Roach values and by WIMS with non $1 / v$ cross sections, it is found that the survival probability is strongly dependent on the initiating energy, particularly in the neighbourhood of resonances. We have also shown that the survival probability obtained from using a 
one group averaged cross section leads to a fission spectrum averaged value of the survival probability, which is in reasonable agreement with the average cross section value.

These results strengthen the validity of using the point model averaged over energy for practical problems in the thermal region. Further work using data sets for more realistic situations involving resonances in the slowing down region would be valuable.

\section{Acknowledgements}

J. E. M. Saxby would like to acknowledge the support of EPSRC under their industrial doctorate (EngD) programme as well as industrial support from the Atomic Weapons Establishment (AWE), Aldermaston, United Kingdom.

M. D. Eaton would like to acknowledge the support of the EPSRC through their support of the grant: Adaptive hierarchical radiation transport methods to meet future challenges in reactor physics (EPSRC Grant No.: EP/J002011/1).

M. M. R. Williams is grateful to Professor Imre Pázsit for some very helpful insights regarding this problem. 


\section{Bibliography}

K. ALMENAS \& R. LEE (1992) "Nuclear Engineering: An Introduction", Springer Verlag, 2014 (softcover reprint of the original 1st Edition 1992).

AMEC FOSTER WHEELER (2014), "WIMS: A Modular Scheme for Neutronics Calculations", User Guide for Version 10 Issue 1, (The ANSWERS Software Package), ANSWERS/WIMS/REPORT/014, Amec Foster Wheeler, Winfrith, Dorset, October 2014

E. ANDERSON, Z. BAI, C. BISCHOF, S. BLACKFORD, J. DEMMEL, J. DONGARRA, J. DU CROZ, A. GREENBAUM, S. HAMMARLIN, A. McKENNEY \& D. SORENSE (1999) "LAPACK Users" Guide", Third Edition, Society for Industrial and Applied Mathematics, Philadelphia

M. S. BARTLETT (1955) "An Introduction to Stochastic Processes", Cambridge University Press

G. I. BELL (1963) "Probability distribution of neutrons and precursors in a multiplying assembly", Annals of Physics, 21, 243

G. I. BELL (1965) "On the Stochastic Theory of Neutron Transport", Nuclear Science and Engineering, 21, 390

G. E. HANSEN \& W. H. ROACH (1961) "Six and Sixteen Group Cross Sections for Fast and Intermittent Assemblies", Los Alamos Scientific Laboratory, LAMS-2543

G. E. HANSEN (1960) "Assembly of fissionable material in the presence of a weak neutron source", Nuclear Science and Engineering, 8, 709

H. HURWITZ, D. B. MacMILLAN, J. H. SMITH \& M. L. STORM (1963) "Kinetics of Low Source Start-Ups: Part I", Nuclear Science and Engineering, 15, 166

A. JONSSON (1963) "THESEUS - A One Group Collision Probability Routine for Annular Systems", AEEW-R 253, United Kingdom. Atomic Energy Establishment (UKAEA), Winfrith.

R. E. MacFARLANE, D. W. MUIR, R. M BOICOURT, A. C. KAHLER (2012) "The NJOY Nuclear Data Processing System, Version 2012", Los Alamos National Laboratory, LA-UR-12-27079

NUCLEAR ENERGY AGENCY (2000) "The JEF-2.2 Nuclear Data Library", AEN NEA, JEFF Report 17 
L. PÁL (1958) "On the Theory of Stochastic Processes in Nuclear Reactors", Supplemento al volume VII, Series X, del Nuovo Cimento, N. 1, 25.

L. PÁL (1962) "Statistical Theory of the Chain Reaction in Nuclear Reactors: Parts I, II, III", Acta Physica Hungaria, English Translation by V. Shibayev, Atomic Energy Authority Report, NP-TR-951 (HaP 31981), Harwell 1962

I. PÁZSIT \& L. PÁL (2008) "Neutron Fluctuations: A Treatise on the Physics of Branching Processes", Elsevier, Amsterdam

M. J. ROTH (1974), "Resonance Absorption in Complicated Geometries" AEEW-R 921, United Kingdom. Atomic Energy Establishment, Winfrith.

M. M. R. WILLIAMS (1966) "The Slowing Down and Thermalization of Neutons", North Holland Publishing Company, Amsterdam

T. F. WIMETT, R. H. WHITE, W. R. STRATTON \& D. P. WOOD (1960) "Godiva II an unmoderated pulse irradiation reactor", Nuclear Science and Engineering, 8, 691 